\title{
Wavelet de-noising technique applied to the PLL of a GPS receiver embedded in an observation satellite
}

\author{
Dib Djamel Eddine \\ Laboratory of Telecommunications and Digital Signal \\ Processing Department of Electronics \\ Djillali Liabes University \\ Sidi Bel Abbes, Algeria.
}

\author{
Djebbouri Mohamed \\ Laboratory of Telecommunications and Digital Signal \\ Processing Department of Electronics \\ Djillali Liabes University \\ Sidi Bel Abbes, Algeria.
}

\author{
Taleb Ahmed Abddelmalik \\ Laboratory of automatics, Mechanical and Computer Science \\ Valenciennes University. \\ France.
}

\begin{abstract}
In this paper, we study the Doppler effect on a GPS(Global Positioning System) on board of an observation satellite that receives information on a carrier wave L1 frequency $1575.42 \mathrm{MHz}$.We simulated GPS signal acquisition. This allowed us to see the behavior of this type of receiver in AWGN channel (AWGN) and we define a method to reduce the Doppler Effect in the tracking loop which is wavelet de-noising technique.
\end{abstract}

Keywords-GPS; Doppler frequency; PLL; wavelet packet denoising;

\section{INTRODUCTION}

A GPS (Global Positioning System) is a geo-localization system operates globally .It includes three segments: Space, Control, user.. GPS signals are transmitted on one frequency, called L1, which contains the code acquisition called «coarse" (C / A), and the various navigation messages $(\mathrm{L} 1=1575.42 \mathrm{MHz})$. Firstly we define the satellite of observation, it belongs to the LEO satellites. Low earth orbit is defined as an orbit within a locus extending from the earth's surface up to an altitude of $2000 \mathrm{Km} \mathrm{[1].}$

Attributing to their high speeds, data transmitted through LEO is handed off from one satellite to another as satellites generally move in and out of the range of earth-bound transmitting stations. Due to low orbits, transmitting stations are not as powerful as those that transmit to satellites orbiting at greater distances from earth's surface.

As LEO orbits are not geostationary, networks of satellites are required to provide continuous coverage. In our case the average of altitude of satellite of observation is $700 \mathrm{~km}$. The period of an observation satellite is 101 minutes [2], than we can compute the velocity of the satellite of observation:

Vob $=\operatorname{Rob} \times d \theta / d t=(700+6368) \mathrm{km}=7330 \mathrm{~m} / \mathrm{s}$

The period of a GPS satellite is $11 \mathrm{~h}, 58 \mathrm{~min}, 2.05 \mathrm{~s}$ [3], as above we compute the velocity of the satellite GPS:
Vgps $=\operatorname{Rgps} \times d \theta / d t=26560 \mathrm{~km}=3874 \mathrm{~m} / \mathrm{s}$

\section{DOPPLER EFFECT}

The change in frequency observed when there is a relative movement between the source and the observer is called the Doppler Effect. It can be given by:

$$
f_{d}=\beta f_{e}
$$

Where:

$f_{d}$ : Doppler frequency

$f_{e}$ : Transmission frequency, and

$$
\beta=\mathrm{Vd} / \mathrm{C}
$$

$\mathrm{Vd}$ is the velocity which causes the Doppler and $\mathrm{C}$ is the celerity.

We have to give extreme cases of the shift Doppler in an observation satellite and it is given by:

$\overrightarrow{v S}=\overrightarrow{V o b}+\overrightarrow{V G P S}$

$v s=\sqrt{V o b^{2}+V G P S^{2}-2 V o b V G P S \cos \theta}$.

(6) Is Generalized Pythagorean relationship where $\theta$ is the angle between two vectors of velocities of the two satellites (GPS and observation).

$\mathrm{v}_{\mathrm{s}}$ is the relative velocity between the satellite GPS and the satellite of observation. The maximum speed between the satellites is when the two satellites are in opposition and the minimum speed is when the two satellites are in the same direction.

Because:

if $\theta=0(\cos \theta>0), \mathrm{Vs}$ achieves its minimum value (the two satellites are in the same direction) 
And if $\theta=\pi \quad(\cos \theta<0)$ Vs awaits its maximum value (the two vectors are in opposite directions).

Therefore

Vs $\min =7330-3874=3456 \mathrm{~m} / \mathrm{s}$.

Vs $\max =7330+3874=11204 \mathrm{~m} / \mathrm{s}$.

Now we define:

$$
V d=\frac{v \operatorname{srecos} \theta}{\sqrt{r e^{2}+r s^{2}-2 r e r s \sin \theta}}
$$

The velocity $\mathrm{Vd}$ which causes the Doppler with:

Vs: relative velocity between the two satellites re: the radius of rotation of the satellite observation rs: the radius of rotation of the GPS satellite

$\theta$ : Is shown in Figure 1:

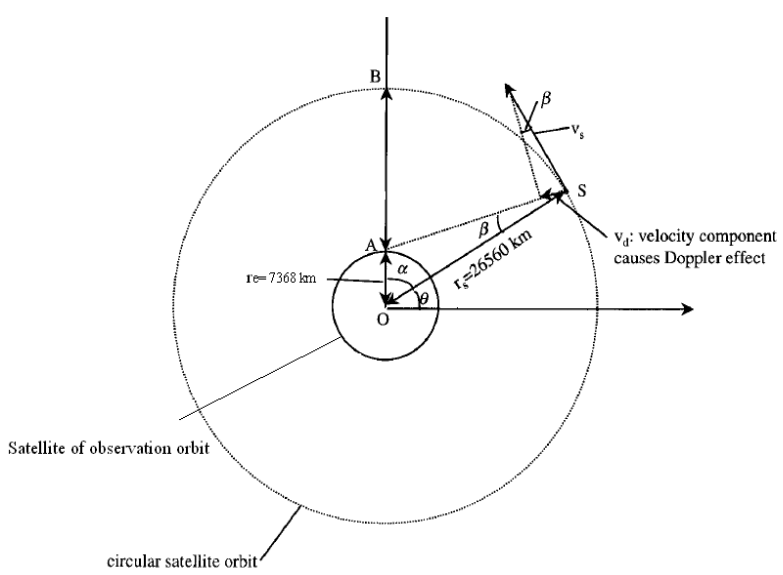

Figure 1. Doppler frequency caused by the velocities of the two satellites.

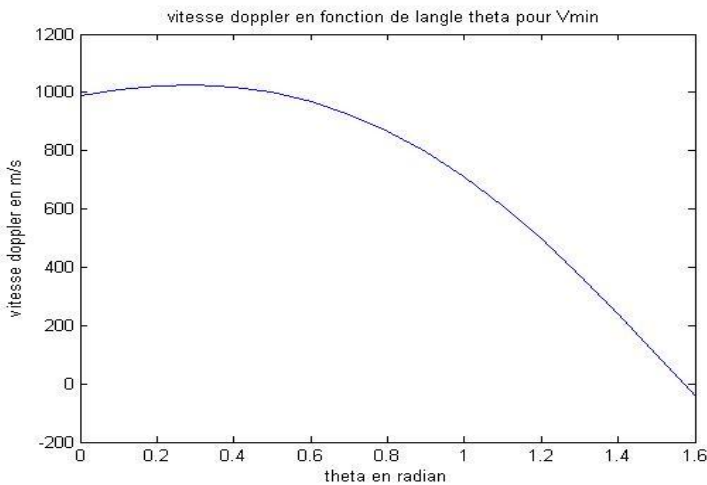

Figure 2. Doppler velocity vs. angle theta at Vs min.

We draw the equation of $\mathrm{Vd}$ with the two value of $\mathrm{Vs}$.

After computing we have

$\mathrm{Vd} \min =1025 \mathrm{~m} / \mathrm{s}$
$\mathrm{Vd} \max =3135 \mathrm{~m} / \mathrm{s}$

Which give two shift Doppler

In the first case $\mathbf{F d} \mathbf{= 5 . 4 K h z}$.

In the second case $\mathbf{F d}=\mathbf{1 6 . 5 K h z}$.

In the first case we observe that it likes a shift Doppler in a GPS in the earth and its graph of acquisition is as shown in figure 4.

In the second case the process of acquisition have a lot of noise because the large bandwidth applied on the PLL filter.

In this section we define firstly the principle of a PLL; then

we give an idea on the wavelet de-noising technique and how we can apply this technique in a PLL.

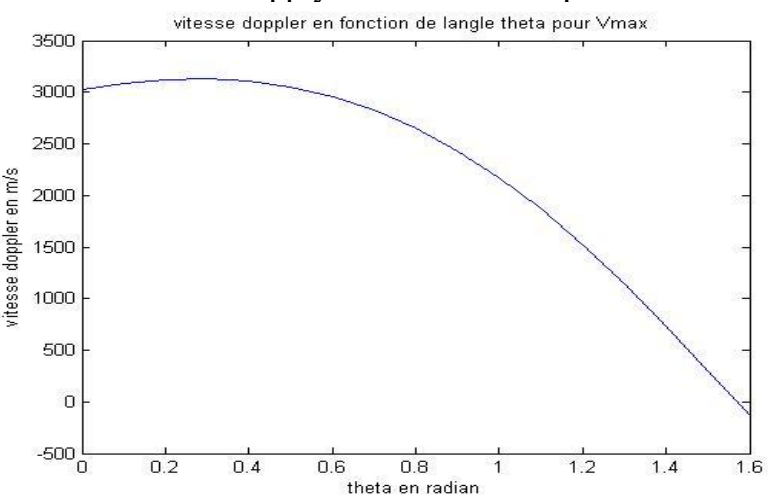

Figure 3. Doppler velocity vs. angle theta at Vs max.

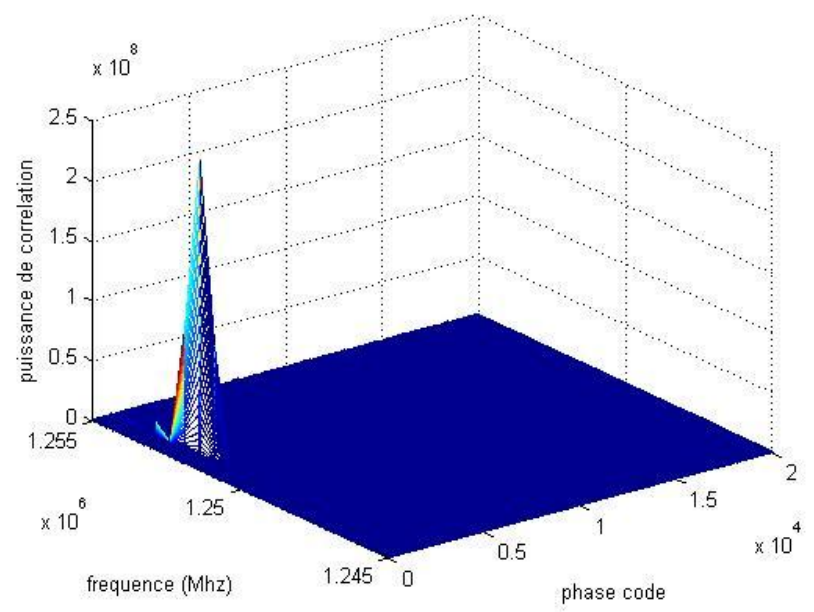

Figure 4. Acquisition of GPS signal at $\mathrm{Fd}=5.4 \mathrm{kHz}$. 


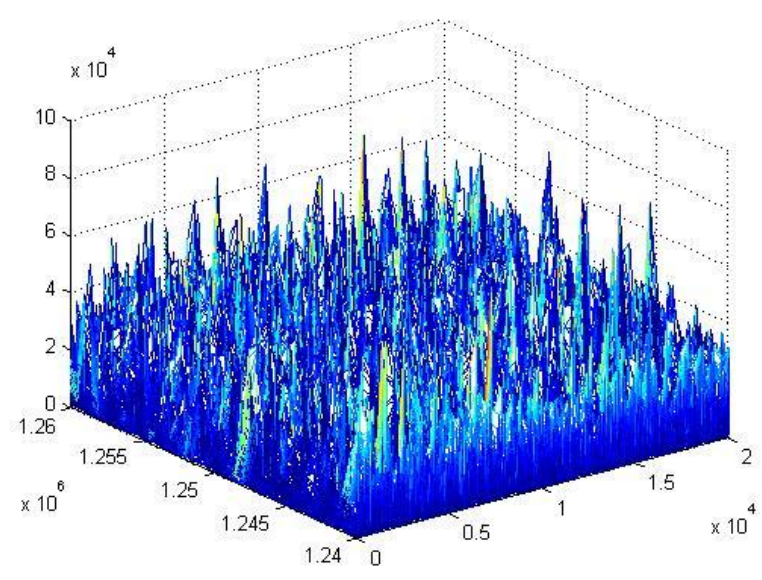

Figure 5. Acquisition of GPS signal at $\mathrm{Fd}=16.5 \mathrm{MHz}$.

\section{BASIC PRINCIPLE OF PlL}

A PLL is a control loop which synchronizes its output signal $\mathrm{u}_{0}(\mathrm{t})$ (generated by a voltage controlled oscillator) with a reference or input signal $\mathrm{u}_{\mathrm{i}}(\mathrm{t})$ in frequency as well as in phase. The PLL generates a control signal which is related to the phase error to control VCO to generate the signal frequency which will be closer to the input signal, until the output frequency of a PLL is exactly same as the input signal and the error signal $\mathrm{u}_{\mathrm{d}}(\mathrm{t})$ between the oscillator's output signal and the reference signal is zero, or remains constant .In such a control mechanism ,the PLL always adjusts the phase of the output signal to lock to the phase of the reference signal [5].A typical PLL block diagram is shown in figure 6 .It consists of three basic function components :a discriminator or a phase detector (PD), a loop filter (LF) and a voltage controlled oscillator (VCO).LF is used to filter the result from the PD and generate the control signal $\mathrm{u}_{\mathrm{f}}(\mathrm{t})$ to control the $\mathrm{VCO}$ to generate the output signal.

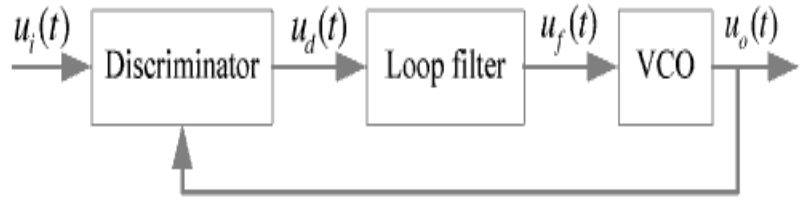

Figure 6. A typical PLL block diagram

\section{Principle Of Wavelet Packet De-Noising}

Signal $f(t)$ can be decomposed into coefficients $\left\{\alpha_{\lambda}(t)\right\}$ which are on another space based on the wavelet packet basis .The signal $f(t)$ can be expressed by the linear superposition of these coefficients:

$f(t)=\sum_{\lambda \epsilon A} a_{\lambda} \beta_{\lambda}(t)$

Where $\left\{\beta_{\lambda}(t)\right\}$ are the basis of the other space .We can abstract the characteristic of the signal from the coefficients $\mathrm{a}_{\lambda}$. So the processing of the signal can be replaced by the processing of $a_{\lambda}$ [6].In this paper ,the wavelet packet decomposition was used.
The wavelet packets transform the signal in time domain into the coefficients in the inner product space of wavelet packet. Define the following notation: $\varphi(\mathrm{x})$ is an scaling function and $\phi(x)$ is the corresponding wavelet function, , $\left\{V_{k}\right\}$ is a multi-resolution space ,also called scale space generated by $\varphi(\mathrm{x}) \cdot\left\{\mathrm{W}_{\mathrm{k}}\right\}$ is a wavelet space generated by $\phi(\mathrm{x}), \mathrm{W}_{\mathrm{k}-1}$ is an fill space differences between $V_{k-1}$ and $V_{k}$, so Lévesque space $\mathrm{L}^{2}(\mathrm{R})$ can be decomposed as [7]:

$$
\begin{gathered}
L^{2}(R)=\cdots \oplus W_{-2} \oplus W_{-1} \oplus W_{0} \oplus W_{1} \oplus W_{2} \\
\bigoplus \ldots
\end{gathered}
$$

And $\left\{2^{\frac{\mathrm{k}}{2}} \phi\left(2^{\mathrm{k}} \mathrm{t}-\mathrm{l}\right): \mathrm{l} \in \mathrm{Z}\right\}$ is a group of bases of $\mathrm{W}_{\mathrm{k}}$. wavelet packet transform can be carried out by followed ways:

Define a sequence of functions as follows [8] :

$$
\left\{\begin{array}{c}
\varphi_{2 n}(x)=2^{j / 2} \sum_{k} h_{k} \varphi_{n}\left(2^{j / 2}-k\right) \\
\varphi_{2 n+1}(x)=2^{j / 2} \sum_{k} g_{k} \varphi_{n}\left(2^{j / 2}-k\right)
\end{array}\right.
$$

Where $\mathrm{j}, \mathrm{k} \in \mathrm{Z}$ ( $\mathrm{Z}$ is an integer set) and $\mathrm{j}$ is a scale parameter is time or location parameter $\epsilon \mathrm{N}$ ( $\mathrm{N}$ is a non -negative integer set), $\mathrm{h}_{\mathrm{k}}$ is a low -pass filter coefficient, $\mathrm{g}_{\mathrm{k}}$ is a high -pass filter coefficient .moreover $\left\{\mathrm{h}_{\mathrm{k}}\right\}$ and $\left\{\mathrm{g}_{\mathrm{k}}\right\}$ are a group of conjugate mirror filters their relationships are as followed :

$\sum_{n \in Z} h_{n-2 k} h_{n-2 l}=\delta_{k, l} \sum_{n \in Z} h_{n}=\sqrt{2} g_{k}=(-1)^{k} h_{l-k}$

Then two -scale equations of wavelet packet transform can be achieved:

$\left\{\begin{array}{l}\varphi_{2 n}(x)=\sqrt{2} \sum_{k} h_{k} \varphi_{n}(2 x-k) \\ \phi_{2 n}(x)=\sqrt{2} \sum_{k} g_{k} \varphi_{n}(2 x-k)\end{array}\right.$

The signal can be expressed by the following wavelet packet bases function:

$f(t)=\sum_{n, j} C_{n}^{j}(k) 2^{\frac{k}{2}} \phi_{n}\left(2^{\frac{k}{2}} t-j\right)$.

Where:

$$
C_{n}^{j}(k)=2^{\frac{k}{2}} \int_{R} f(t) \phi_{\mathrm{n}}\left(2^{\frac{k}{2}} t-j\right) d t
$$

Based on the theory of local maxima value of the wavelet transformation, the characteristic information is concentrated in a few coefficients .So, de-noising process can be done by saving the character coefficients and threshold other coefficients.

After threshold, the modified coefficients can be used to reconstruction of signal. The reconstruction formulation of a discrete signal is done by [8]:

$$
C_{m}^{j}(k)=\sum_{n} \bar{h}_{k-2 n} C_{2 m}^{j+1}(n)+\sum_{n} \bar{g}_{k-2 n} C_{2 m+1}^{j+1}(n)
$$

$\bar{h}_{k-2 n}$ and $\bar{g}_{k-2 n}$ can be obtained by reversing order of $h_{k-2 n}$ and $g_{k-2 n}$.

Wavelet packet decomposition can decompose the signal to different frequency bands in different levels. If we have sufficient decomposed levels and data samples the beginning and the ending of a frequency band can be acquired [6]. 
In general wavelet packet de-noising is done in the following steps :

Firstly, decompose the signal based on the selected wavelet packet basis. The signal is decomposed into several layers of wavelet packet coefficients as a tree [6].

The structure of the tree is as figure 7 :

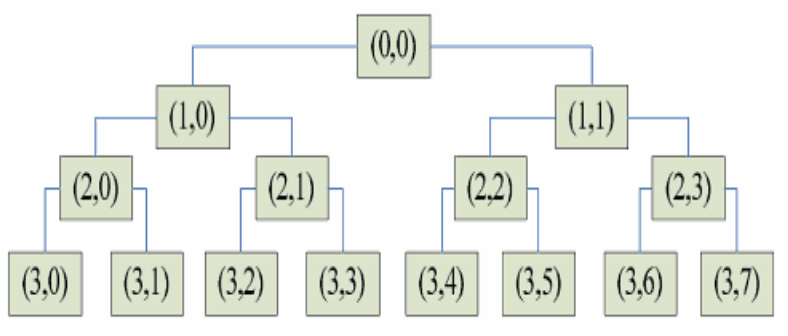

Figure 7.

Secondly, compute the best tree based on the entropy of Shannon, which is computing the best wavelet packet basis.

Thirdly, a threshold is computed and then a soft-threshold is applied to the coefficients. The threshold can be calculated as follows [8]:

$\lambda=\sigma \sqrt{2 * \log _{2} N}$

There are two kinds of threshold functions, hard threshold and soft threshold functions. In this paper, soft threshold function was selected .It is defined as [8]:

$\widehat{W}_{j, k}\left\{\begin{array}{r}\operatorname{sign}\left(W_{j, k}\right)\left(\left|W_{j, k}\right|-\lambda\right),\left|W_{j, k}\right| \geq \lambda \\ 0 \quad,\left|W_{j, k}\right|<\lambda\end{array}\right.$

Fourthly, signal reconstruction is performed using the modified detail coefficients.

\section{APPLYING WAVELET PACKET DE-NOISING FOR A PLL}

The purpose of applying the wavelet packet de-noising technique in the PLL is to reduce the noise level before the loop filter the block diagram is as figure 8 .

The main function of the wavelet packet de-noising is to reduce the noise level within the bandwidth of the loop filter the loop filter could filter out the noise beyond the bandwidth of the loop filter ,but the noise within the bandwidth still could pass through and affect the NCO tracking performance.

It should be noted that the wavelet packet de-noising technique can only reduce the noise level rather than eliminate the noise totally.
The remaining noise still can affect the NCO tracking performance but at a lower level[9].

The wavelet packet de-noising technique may cut off some useful signals which are smaller than the threshold .This disadvantage will make the PLL spend more time to lock due to the loss of some control signals and output from PLL distorted. However the decrease of the noise will produce smaller phase error that will help the PLL to maintain locked and smooth the output [6].

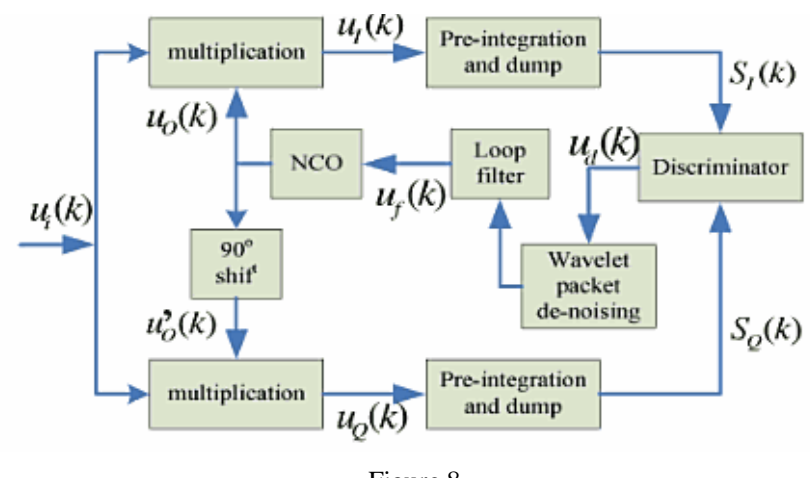

Figure 8.

\section{TEST AND Results}

In our case we have $16.5 \mathrm{KHZ}$ such as a Doppler frequency and we will see the behavior of the loop of tracking with the both configuration

1-without applying of wavelet packet de-noising

2-with applying of wavelet packet de-noising

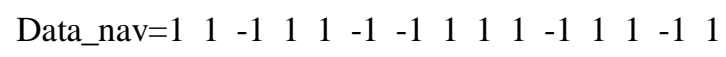

A. without applying of wavelet packet de-noising:

We will see the signal at the input of loop filter (this signal will command the NCO) and also the bits after tracking.

We observe that because the lots of noise in the signal at the input of loop filter we have bad results (weak signal) so we cannot extract the bits.

\section{B. with applying of wavelet packet de-noising:}

We apply the wavelet packet de-noising just before the loop filter and we have these results:

This method de-noise the signal to have good performance especially when we have a lot of noise because the higher Doppler frequency and we observe that the bits tracked are similar to the original signal GPS. 


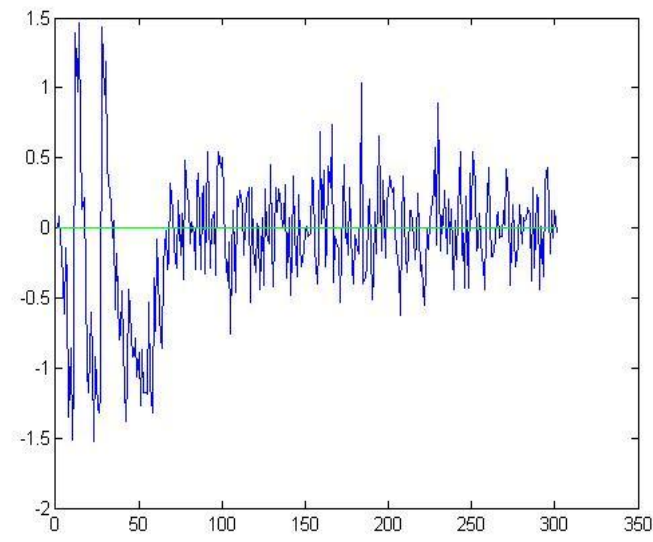

Figure 9. The signal at the input of loop filter without applying wavelet packet de-noising

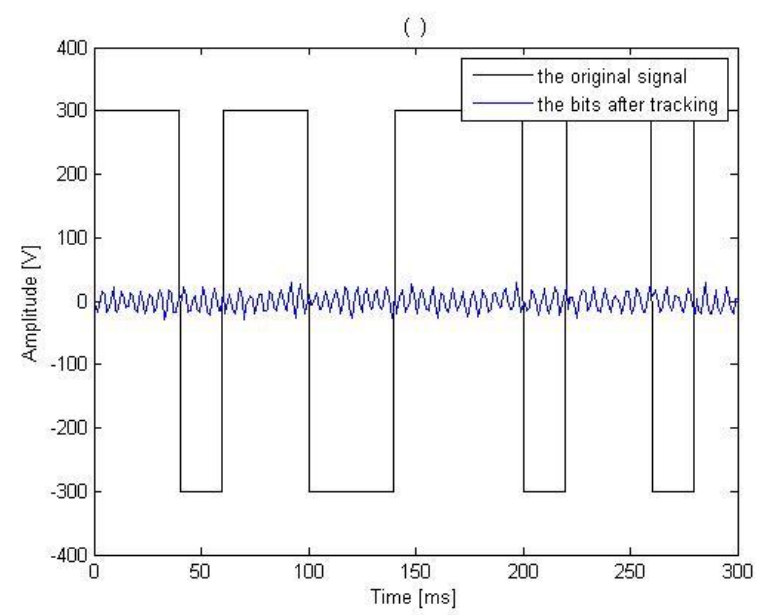

Figure 10. The bits after tracking without applying wavelet packet de-noising.

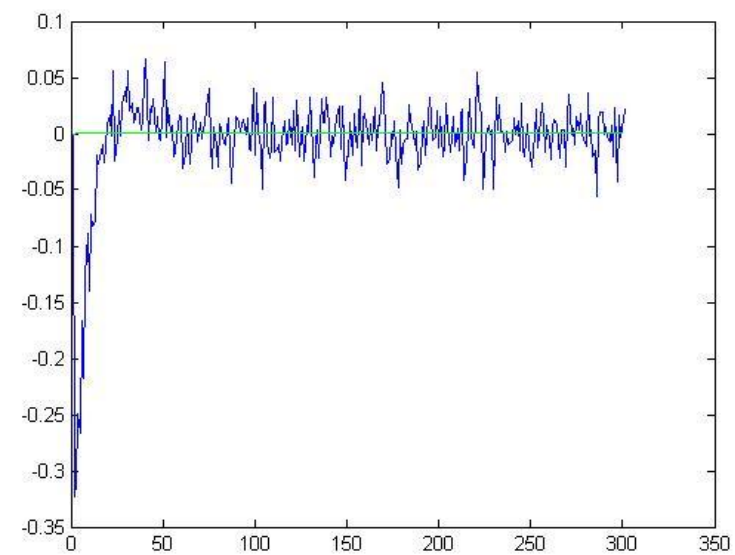

Figure 11. The signal at the input of loop filter with applying wavelet packet de-noising

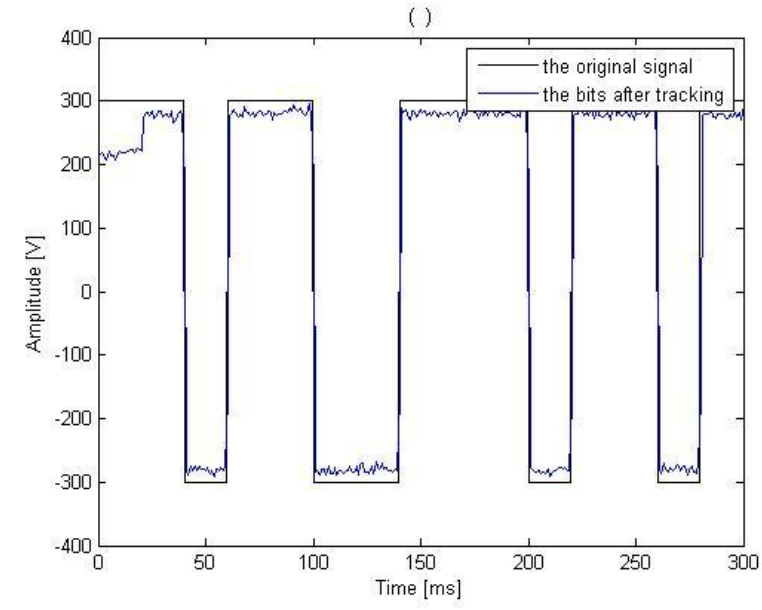

Figure 12. The bits after tracking with applying wavelet packet de-noising

\section{CONCLUSION}

Applying the wavelet packet de-noising technique in the PLL helps to reduce the noise within the bandwidth of the loop filter and therefore, a less noisy tracking performance can be obtained from the NCO, we observe that by using this method we have less error in the bits in the end the process of tracking .than the ordinary method that do not use the wavelet packet de-noising.

This method is recommended in the GPS embedded in the satellite of observation because the high effect of Doppler frequency.

\section{REFERENCES}

[1] IEEE journal on selected areas in communications .vol.13 no.2 February 1995

[2] www.astronoo.com/satelliteobservation

[3] Fundamentals of Global Positioning System Receivers: A Software Approach James Bao-Yen Tsui Copyright @ 2000 John Wiley \& Sons, Inc. Print ISBN 0-471-38154-3 Electronic ISBN 0-471-20054-9 page $37-49$

[4] Gardner, F.M: Phase lock techniques, 3rd demijohn Wiley and sons, Inc, USA (2005)

[5] Qian H-m,Ma,J-c,Li,Z-y :fiber optical gyro de-noising based on wavelet packet soft -threshold algorithm .journal of chinese Inertial technology 15(5),602-605(2007)

[6] daubechies ,I :orthonormal bases of compactly supported wavelets .commun.pure appm.41,909-996(1998)

[7] peng,Y,weng ,X.H :thresholding -based wavelet packet methods for doppler ultrasound signal denoising in :IFMBE proceedings, APCMBE 2008 ,vol 19,pp.408-413(2008)

[8] Lian,P,Lachapelle G,Ma,C.L :Improving tracking performance of PLL in high dynamics applications ION.NTM,1042-1058(2005). 